\title{
Universal maximally entangled states
}

\author{
M. Revzen
}

Received: 29 May 2014 / Accepted: 20 August 2014 / Published online: 23 September 2014

(C) Chapman University 2014

\begin{abstract}
Two $(i=1,2) d$-dimensional $(d=$ prime, $\neq 2)$ particles are accounted for via universal maximally entangled states (MES) bases. The bases are labeled by the particles' collective ("center of mass" and "relative") coordinates in a phase space like formalism. Schmidt's decomposition represents MES via $d$-terms of single particle product states that form a 'line' in a $d^{2}$ array of points each designating a single particles product states. Correspondingly, a 'line' in a $d^{2}$ array of phase space like points designating a $d^{2}$ orthonormal MES, represents a two particles product state.
\end{abstract}

Keywords Maximally entangled states $\cdot$ Mutually unbiased bases $\cdot$ Schmidt decomposition

\section{Introduction}

The most remarkable attribute of quantum mechanics (QM) is its association of physical processes with linear relations among probability amplitudes. A striking consequence of this is the appearance of entangled states [10]. These states, introduced by Einstein, Podolsky and Rosen (EPR) [7], were shown to be intimately involved in almost all subtle characteristics of QM which even today defies comfortable understanding [9].

The almost complete dominance of these states in studies of the foundation of QM studies was instigated by Bohm who formulated the EPR considerations within a finite dimensional Hilbert space: he considered spin states and orientations rather than position and momenta which have continuous values. Bell's analysis $[6,8]$ of the EPRBohm study revealed a non-locality facet that may reside in entangled particle's pair correlation. Introduction of a basis of orthonormal maximally entangled states [3] in this context encouraged the formulation of protocols for teleportation [5], entangling QM with information theory which is, arguably, the central theme of present day investigations of QM foundations.

In this work, we study maximally entangled states (MES) of two d-dimensional particles (systems). Our study is confined to $d$ a prime $\neq 2$ since for these dimensionalities the mathematics is simplest - thus though extensions to $d$ all primes and power of primes are possible [16-19,22,26] it requires a more sophisticated mathematics while, we judge, not enhancing physical transparency. We begin with a brief review of an information theoretic definition [23,24] of Maximally Entangled States (MES) (Sect. 2) [11]. In Sect. 3 we show that every MES may be viewed

M. Revzen ( $\square)$

Department of Physics, Technion-Israel Institute of Technology, 32000 Haifa, Israel

e-mail: revzen@physics.technion.ac.il 
as a product state in appropriately selected collective coordinates. Thus, whereas the strong correlation between the entangled particles is studied extensively, the present study considers the independence between "collective" variables implied thereby. Such a representation of MES as a product state (in the collective variables) is used to conveniently define an orthonormal MES basis for the $d^{2}$ dimensional Hilbert space. These are product states of the two particles/systems "center of mass" like and "relative" coordinates: the state is specified with phase space like parameters with the $d^{2}$ phase space like points parameterizing the $d^{2}$ dimensional orthonormal MES basis of the whole Hilbert space. The next section, Sect. 4, contains (finite) geometry notions $[14,29]$ for the $d^{2}$ square array of phase space like points: We consider states formed by sums of states underpinned with points $(q, p)$ of the phase space (which are MES) that lie on a straight line. We show that the resultant states are (product of) single particle Mutually Unbiased Bases (MUB) states, revealing, thereby, a novel scheme for the construction of MUB states. Furthermore, as is given in Sect. 5, it discerns inversion of the Schmidt [4,12] diagonal form of MES.

We add four appendices. The first, Appendix A, reviews [29] the definition of "tilde" states used in the discussion of the "universal" form of MES in Sect. 2. Appendix B elaborates state relabeling issue which allows close correspondence between the labeling of the isomorphic Hilbert spaces of the two particles. Appendix C details our definition of collective coordinates [30-32] and indicates the source of the nomenclature "center of mass" and "relative" coordinates, while Appendix D provides a brief review for the particular Mutually Unbiased Bases (MUB) used in the text.

\section{Maximally entangled states (MES) and line states}

Information theoretic approach to entanglement is considered in [23,24]. The present study deals with special case thereof: maximal entanglement of two equi-dimensional $(d)$ particles in a pure state and their MES bases. A complete orthonormal basis, for $d=2$, of maximally entangled states (MES) was introduced in [3]; an in-depth study of these bases is due to Fivel [11]. Further works dealing with MES bases were published much later: [25,26,29]. We shall follow [11,29] in the main.

We define MES in close analogy with [11] (This definition is, in essence, a special case of the one given by $[23,24])$.

Definition: MES of two $d$-dimensional particles (or systems-we use the terms interchangeably) is a pure state, $\mid u>$, implying that the probability of having particle $i(i=1,2)$ in a arbitrary single particle state $\mid \alpha>$ is independent of $i$ and $\alpha$.

It is a mathematically proven result that every two particles state is expressible in Schmidt decomposed $[4,12]$ sum of mutually orthogonal terms each involves product state of the two particles. One notes that the Schmidt decomposition of MES is, of necessity, of the form

$|u(1,2)\rangle=\frac{1}{\sqrt{d}} \sum_{m=0}^{d-1}|m, b\rangle_{1}\left|m-c, b^{\prime}\right\rangle_{2} ; \quad c=0,1, \ldots d-1$.

The sum, in Eq. (1), defines two orthonormal bases, $b$ and $b^{\prime}$ - the summation $(\bmod [d])$ includes all $d$ (orthonormal) states for either particle. This is necessary for if any member $m$ of either basis were missing the probability of having the relevant particle in that state will be nil , contrary to the MES definition. Thus the expansion specifies two orthogonal bases: one for each of the particles (Notational note: particles are designated numerically while quantum states by given letters. Thus $|u(1,2)\rangle$ have the numbers, 1,2 , refer to the two particles while $u(q, p)\rangle$ denotes that particle 1 in a state labeled by $q$ and 2 in $p$. When possible confusion could arise the specification is explicit). The form, Eq. (1), is sufficient for a MES of two $d$-dimensional particles. This may be verified upon partial tracing of such state with respect to either particle coordinates leaves unity as the resultant density operator of its mate: Thus the probability of either particle is in any single particle state is $1 / d$, e.g., the probability of particle 2 is 
in the (single particle) state $|\alpha\rangle$

$P(2, \alpha)=\operatorname{tr}|u\rangle\langle u \mid \alpha\rangle_{2}\langle\alpha|=\frac{1}{d}$

Thus, we have shown that the definition of MES as given above is both necessary and sufficient to imply that every MES can be expressed in the form of Eq. (1).

It is noteworthy that each MES allows a convenient definition for a MES basis for the entire two $d$-dimensional particles' Hilbert space. Thus the $d^{2}$ states generated from such state,

$\left|u_{q, p}^{b, b^{\prime}}\right\rangle=\frac{1}{\sqrt{d}} \sum_{m}|m ; b\rangle_{1} \omega^{-m p}\left|m-q ; b^{\prime}\right\rangle_{2} ; \quad q, p=0,1, \ldots d-1, m-q=m+(d-q) \operatorname{Mod}[d] ; \omega=e^{i \frac{2 \pi}{d}}$.

are, as can be verified by direct substitution, orthonormal

$\left\langle u_{q, p}^{b, b^{\prime}} \mid u_{q^{\prime}, p^{\prime}}^{b, b^{\prime}}\right\rangle=\delta_{q, q^{\prime}} \delta_{p, p^{\prime}}$,

and thence forms a complete orthonormal MES basis for the Hilbert space. We shall find it convenient to refer to a product state, e.g. $|m ; b\rangle_{1}\left|n ; b^{\prime}\right\rangle_{2}$ as a "point" in a square $d \times d$ array whose $x$ (discrete) coordinate is $m$ and the $y$ coordinate is $n$. Thus we may view the state in Eq. (3) as a "line" state-it runs over $d$ "points" (the summation is modular, mode $[d]$ ) underpinning the line $m(n)=n+q \operatorname{Mod}[d]$. Schmidt decomposition is seen thereby to be a line state expression for MES.

An appropriate relabeling (cf. Appendices A, B) allows the rewriting of Eq. (1) in a "universal", i.e. basis independent, form

$|u(1,2)\rangle \rightarrow|\mathcal{R}\rangle=\frac{1}{\sqrt{d}} \sum_{m}|m ; b\rangle_{1}|\tilde{m} ; \tilde{b}\rangle_{2}$.

The "tilde" state is defined in Appendix A where the universality is demonstrated [22,30-32]. We may now use the basis independence of the state $|\mathcal{R}\rangle$ to rewrite Eq. (1) as

$|u(1,2)\rangle \rightarrow|\mathcal{R}\rangle=\frac{1}{\sqrt{d}} \sum_{m}|m ; \ddot{0}\rangle_{1}|\tilde{m} ; \ddot{0}\rangle_{2}$,

which in turn may be used to define a complete orthonormal MES basis,

$\Rightarrow|u(q, p)\rangle_{b=0}=\frac{1}{\sqrt{d}} \sum_{m}|m+q ; \ddot{0}\rangle_{1} \omega^{m p}|m-q ; \ddot{0}\rangle_{2} ; q, p=0,1,2 \ldots d-1$,

where bot particles are in the $\mathrm{CB}(b=\ddot{0}$, cf. Appendix $\mathrm{C})$.

\section{Maximally entangled states in collective coordinates}

The collective coordinates, defined in Appendix B, are referred to as "center of mass", $c$, and relative, $r$. Each relates, of course, to its corresponding state enumerator operator, $Z$ and state shifting operator, $X$. The eigenstates of $X$ are the Fourier transforms of those of $Z$. The latter's eigenstates are referred as the CB for the respective collective coordinate: In the following the eigenvalues of $Z$ (for all systems: particles 1, 2 and collective coordinates $c, r$ ) will 
be denoted by $q$ and the basis, $b$, by $\ddot{0}$, those of $X$ by $p$ and the corresponding basis, $b$, is designated by $b=0$; $q, p=0,1, \ldots d-1$. Thus

$Z_{s}|q ; \ddot{0}\rangle_{s}=\omega^{q}|q ; \ddot{0}\rangle_{s} ; \quad s=1,2, c, r ;\langle q ; \ddot{0} \mid p ; 0\rangle=\frac{\omega^{-q p}}{\sqrt{d}}$.

Returning to Eq. (7) and recalling [30-32] and Appendix C the transformation to the collective coordinates is affected by,

$|m ; \ddot{0}\rangle_{1}\left|m^{\prime} ; \ddot{0}\right\rangle_{2}=\left|\frac{m+m^{\prime}}{2} ; \ddot{0}\right\rangle_{c}\left|\frac{m-m^{\prime}}{2} ; \ddot{0}\right\rangle_{r}$.

Utilizing this we rewrite the arbitrary MES, Eq.(7),

$$
\begin{aligned}
|u(q ; p)\rangle_{\ddot{0}} & =\frac{1}{\sqrt{d}} \sum_{m}|m+q ; \ddot{0}\rangle_{1} \omega^{-m p}|m-q ; \ddot{0}\rangle_{2} \\
& =|q ; \ddot{0}\rangle_{r}\left(\frac{1}{\sqrt{d}} \sum_{m}|m ; \ddot{0}\rangle_{c} \omega^{-m p}\right)=|q ; \ddot{0}\rangle_{r}|p ; 0\rangle_{c} .
\end{aligned}
$$

Demonstrating thereby that every (the state considered was arbitrary) MES is a product state in collective coordinates: in the formalism considered the "center of mass" collective coordinate has its value in momentum space (it is the Fourier transform of the $\mathrm{CB}$ ) while the "relative" coordinate has its value in the $\mathrm{CB}$. We have thus that the two $d$-dimensional particles' Hilbert space is spanned by the $d^{2}$ orthonormal phase space like points

$|u(p, q)+\rangle=|p ; 0\rangle_{c}|q ; \ddot{0}\rangle_{r}=Z_{c}^{-p} X_{r}^{q}|0 ; 0\rangle_{c}|0 ; \ddot{0}\rangle ; \quad q, p=0,1, \ldots d-1$.

One readily verifies that these are MES:

$|p ; 0\rangle_{c}|q ; \ddot{0}\rangle_{r}=\frac{1}{\sqrt{d}} \sum_{m}|m ; \ddot{0}\rangle_{c} \omega^{-m p}|q ; \ddot{0}\rangle_{r}=\frac{1}{\sqrt{d}} \sum_{m}|m+q\rangle_{1} \omega^{-m p}|m-q\rangle$.

The formalism suggests the definition of a conjugate "phase space" like basis, $|u(p, q) ;-\rangle$,

$|u(p, q) ;-\rangle \equiv|q ; \ddot{0}\rangle_{c}|p ; 0\rangle_{r}=X_{c}^{q} Z_{r}^{-p}|0 ; \ddot{0}\rangle_{c}|0 ; 0\rangle_{r}$.

Thus MES have a lattice phase space structure [11] wherein a lattice point $(q, p)$ is realized by collective coordinates product state $(q, p) \Leftrightarrow|q ; \ddot{0}\rangle_{c}|p ; 0\rangle_{r}$. Studies of dynamically induced "hopping" on this lattice [11] generated by unitary operator $U(t)$ that causes the two particles to evolve such that at discrete times, $t=0,1,2 \ldots$, their state will coincide with a lattice site is direct within the collective coordinates formulae: $U(t)$ is a simple product of powers of the collective coordinates operators, $X_{c}, Z_{c} ; X_{r}, Z_{r}$. e.g.,

$$
\begin{aligned}
X_{c}^{t=2} X_{r}^{3(t=2)}|q ; \ddot{0}\rangle_{c}|p ; 0\rangle_{r} & =\omega^{6 p}|q+2 ; \ddot{0}\rangle_{c}|p ; 0\rangle_{r} \\
(q, p) & \Rightarrow \omega^{6 p}(q+2, p) .
\end{aligned}
$$

We now note a remarkable attribute of MES, striking especially in cases where the particles are widely separated: Let us act on one of the particles, e.g., on particle 1, with $\hat{X}_{1}^{2}$. The state of the particle (one in our case) is unaffected: the probability of it being in any single particle $|\alpha\rangle$ remains $1 / d$ - independent of $\alpha$. However, the collective coordinates are affected, will undergo a simple change. Explicitly,

$$
\begin{aligned}
& \hat{X}_{1}^{2} \frac{1}{\sqrt{d}} \sum_{m}|m ; b\rangle_{1}|\tilde{m} ; \tilde{b}\rangle_{2}=\frac{1}{\sqrt{d}} \sum_{m^{\prime}}\left|m^{\prime} ; b\right\rangle_{1} \sum_{m} U_{m^{\prime}, m}^{b}|\tilde{m} ; \tilde{b}\rangle_{2} \\
& =\frac{1}{\sqrt{d}} \sum_{m^{\prime}}\left|m^{\prime} ; b\right\rangle_{1} \sum_{m} U_{\tilde{m}^{\prime}, \tilde{m}}^{\tilde{b}}|\tilde{m} ; \tilde{b}\rangle=\frac{1}{\sqrt{d}} \sum_{m^{\prime}}\left|m^{\prime} ; b\right\rangle_{1}\left|\tilde{m}^{\prime} ; \tilde{b}\right\rangle,
\end{aligned}
$$


i.e., the state remains a MES: either particle may be found with equal probability in any single particle state, i.e., neither particle's expectations values of (any) single particle operator are affected.

We now consider the effect on the collective coordinates state. The universal state representation of the MES, was shown above to imply

$$
\frac{1}{\sqrt{d}} \sum_{m}|m ; b\rangle_{1}|\tilde{m} ; \tilde{b}\rangle_{2}=|0 ; 0\rangle_{c}|0 ; \ddot{0}\rangle_{r}
$$

Thus,

$\hat{X}_{1}^{2} \frac{1}{\sqrt{d}} \sum_{m}|m ; b\rangle_{1}|\tilde{m} ; \tilde{b}\rangle_{2}=\hat{X}_{r} \hat{X}_{c}|0 ; 0\rangle_{c}|0 ; \ddot{0}\rangle_{r}=\hat{X}_{r} \hat{X}_{c} \frac{1}{\sqrt{d}} \sum_{n}|0 ; 0\rangle_{c}|n ; 0\rangle_{r}=|0 ; 0\rangle_{c}|-1 ; \ddot{0}\rangle_{r}$,

i.e., the states collective coordinates are affected.

\section{Mutually unbiased bases and the inversion of Schmidt decomposition}

Mutual unbiased bases (MUB) were introduced by Schwinger [1] as the bases associated with operators of "maximum degree of incompatibility", the information theoretic oriented appellation "Mutually Unbiased Bases" (MUB) that is essentially universal now, was dubbed by Wootters $[18,19]$. Appendix D gives a brief theoretical overview of MUB. MUB, aside from their direct theoretical significance as representatives of the uncertainly among (relevant) observables, are widely used in studies of foundations of quantum mechanics (QM), information and cryptography. Several approaches to their construction are known [16-22,26,29], we present here a new scheme, underscoring their relation to MES [13,29] which may provide new insight on their role in QM.

We have shown above, Eq. (10), that an arbitrary MES $|u\rangle$, defines and thus provides an "origin" for $d^{2}$ orthonormal states, $u(q, p)\rangle=|q ; \ddot{0}\rangle_{c}|p ; 0\rangle_{r} q, p=0,1, \ldots d-1$ (Recall that $\ddot{0}$ dubs the $\mathrm{CB}$, the eigenfunctions of $\bar{Z}$, while 0 (in the vectorial label, dubbed $|p ; 0\rangle_{r}$ ) —eigenfunctions of $\bar{X}$ ). These states now realize points in a $d^{2}$ square array whose rows (lines parallel to the $x$ axis) are enumerated by $p$ and its columns by $q$ (parallel to the $y$ axis). This $d$ by $d$ square will be referred to as "phase space". We define a line, $L$, in this phase space, as the aggregate of $d$ points given by linear relation between $p$ and $q$. E.g. for $d=3$ the line given by the equation, $p$ $=0$, consists of the three points whose coordinates are $(0,0) ;(1,0)$ and $(2,0)$, whereas the line $p=q$ contains the points $(0,0) ;(1,1)$ and $(2,2)$. In our study the points are realized by states. Thus, in the first case above, the three states are $|0 ; \ddot{0}\rangle_{c}|0 ; 0\rangle_{r} ;|1 ; \ddot{0}\rangle_{c}|1 ; 0\rangle_{r} ;|2 ; \ddot{0}\rangle_{c}|2 ; 0\rangle_{r}$. We now define a line state (yet to be normalized) by the sum of its aggregate points. Since a line is determined by two of its points the generic designation of a line state is $\left|L\left[\left(q_{0}, p_{0}\right) ;\left(q_{1}, p_{1}\right)\right]\right\rangle$ (we shall abbreviate the notation below). There are $d+1$ distinct lines emerging from the origin realized by $|0 ; \ddot{0}\rangle|0 ; 0\rangle: d$ of these are given by $p=b q ; \operatorname{Mod}[d] ; b=0,1, \ldots d-1$ and an additional line along the $x$ axis whose line equation is $q=0$, is given by the points $|q ; \ddot{0}\rangle|0 ; 0\rangle ; q=0,1, \ldots d-1$. There are $d$ parallel lines to each of the $d+1$ lines. e.g., for $d=7$, the lines parallel to $p=3 q ; \operatorname{Mod}[7]$ are the $d$ lines given by $p=3 q+s, \operatorname{Mod}[7] ; p, q, s=0,1, \ldots 6$. Another set of parallel lines is $p=s ; s=0,1, \ldots 6$. It is obvious that each of the $d+1$ lines and its $d$ parallels contain exactly all $d^{2}$ points. We label $d$ of these $d+1$ sets by their $b$ value (the set parallel to $q=0$ by $\ddot{0}$ ) i.e. a line is specified by its set $b$ which gives its orientation and $s$ that specifies its position relative to the line of the same orientation that goes through the origin (This labeling will not give rise to any conflict with the previous notation). We now assert that each of these sets is an MUB basis and each of the line state is a product of MUB states, one for each particle. This is the novel construction of the MUB states. We now prove the assertion.

Consider first the simplest case: the line $q=m$ that runs parallel to the $y$ axis at $x=m$, i.e. the line with $b=\ddot{0}$ and $s=m$, this state line $|L(\ddot{0} ; m)\rangle$,(we ignore the normalization) is:

$$
|L(\ddot{0} ; m)\rangle=\sum_{p}|m ; \ddot{0}\rangle_{c}|p ; 0\rangle_{r}=\sum_{p, n}|m ; \ddot{0}\rangle_{c}|n ; \ddot{0}\rangle_{r} \omega^{-n p}=|m ; \ddot{0}\rangle_{c}|0 ; \ddot{0}\rangle_{r}=|m ; \ddot{0}\rangle_{1}|m ; \ddot{0}\rangle_{2}
$$


We used $\sum_{p} \omega^{p n}=d \delta_{n, 0}$ and $n_{1}=n_{c}+n_{r} ; \quad n_{2}=n_{c}-n_{r}$. Thus we got that the line state is a product state of a particle 1 state with its tilde state of particle 2 (note for $b=\ddot{0}$ the tilde state is identical with its mate).

The study thus far indicated that Schmidt decomposition of MES may viewed as expressing the MES as a "line state" made of "points" each of which is a product state in the particle coordinates. The "line state" and thereby the MES it account for was shown to be a product state in appropriately chosen collective coordinates. This suggests considering product state (in the particle coordinates) be related a "line state" in the phase space like square array whose constituent points space $(q, p)$ that underpin product states in collective coordinates. Such an account of particles product state is termed inversion of the Schmidt decomposition. We shall now show that, indeed, summing over phase space points on a straight line, viz. (denoting the line state by $|L(m, b)\rangle$ ),

$|L(m,, b)\rangle=\frac{1}{\sqrt{d}} \sum_{q}|p(q) ; 0\rangle_{c}|q ; \ddot{0}\rangle_{r} ; \quad p(q)=b q+m ; q, p, b, m=0,1, \ldots d-1$,

gives a product state in the single particle coordinates, i.e. invert the Schmidt diagonalization, the line state equals a product of two single particle MUB states:

$$
\begin{aligned}
|L(m,, b)\rangle & =\frac{1}{d} \sum_{q} \sum_{n} \omega^{-n p}|n\rangle_{c}|q\rangle_{r}=\frac{1}{d} \sum_{q} \sum_{n} \omega^{-n(b q+m)}|n+q\rangle_{1}|n-q\rangle_{2} \\
& =\frac{1}{d} \sum_{n_{1}} \sum_{n_{2}}\left|n_{1}\right\rangle_{1}\left|n_{2}\right\rangle_{2} \omega^{-\frac{n_{1}+n_{2}}{2}\left[b\left(n_{1}-n_{2}\right)+m\right]} \\
& =\frac{1}{\sqrt{d}} \sum_{n}|n\rangle_{1} \omega^{\frac{b}{4} n^{2}-\frac{m}{2} n} \frac{1}{\sqrt{d}} \sum_{n}|n\rangle_{2} \omega^{-\frac{b}{4} n^{2}+\frac{m}{2} n}, \\
& \left.\left.=\left|\frac{m}{2} ; \frac{b}{4}\right\rangle\right\rangle_{2}\left|\frac{\tilde{m}}{2} ; \frac{\tilde{b}}{4}\right\rangle\right\rangle_{1} .
\end{aligned}
$$

We used Eqs. $(11,32,33,37)$; the double angular bracket signifies an MUB state, cf. Appendix D:

$|m ; b\rangle\rangle=\frac{1}{\sqrt{d}} \sum_{n}|n\rangle \omega^{b n^{2}-m n}$.

Thus the "line state" — constituting "points", each designating a product collective coordinates states, i.e., MES in particles coordinates - expresses product single particle state, i.e. reversing Schmidt diagonalization. The result yielding product MUB states provides a novel way for obtaining MUB states.

We further note that the two bases Eqs. $(10,12)$ are mutually unbiased:

$\left|\left\langle u\left(q^{\prime} p^{\prime} ;-\right) \mid u(q, p ;+)\right\rangle\right|=\frac{1}{d} ; \forall q, q^{\prime}, p, p^{\prime}$.

This relation provided the means for determining separately the state and the measurement-basis in [27-29].

\section{Conclusions and remarks}

Maximally entangled states (MES) for two $d$-dimensional particles, $d=$ prime $\neq 2$, were defined via slightly modified study by Fivel [11] (and $[23,24]$ ) as a pure two particle state in which the probability of finding particle $i(i=1,2)$ in a single particle state $|\alpha\rangle$ is independent of $i$ and $\alpha$. This underscores an information theoretic, purely quantal, attribute of entanglement noted earlier in [23,24]: stronger quantum correlation among the pair entails weaker individual specification. Thus maximum entanglement randomizes the state of its constituents. Mathematically such a state is a pure two particles state whose Schmidt decomposition [4,12] 
involves a $d$-terms sum of product state containing, with equal weight, full bases of the two $d$-dimensional Hilbert spaces. As such it has a "universal", i.e. basis independent, characterization which has a geometrical interpretation [32].

It was argued that every MES allows natural definition for a MES basis, viz $d^{2}$ orthonormal MES, spanning the two $d$-dimensional particles' Hilbert space wherein the states, forming the basis are viewed as "line states" each being a Schmidt decomposition of a MES.

We then showed that every MES may be expressed as a product state in appropriately chosen collective coordinates denoted by $c$ ("center of mass") and $r$ ("relative"). These are studied in terms of their Schwinger operators, $\hat{Z}_{s},(s=$ $c, r$ ), whose eigenfunctions are the reference basis (dubbed computational basis (CB) almost universally and which we denote by $b=\ddot{0}$ ) and $\hat{X}_{S}$ which is the Schwinger shift operator whose eigenfunctions form the Fourier transform of the $\mathrm{CB}$ (that is denoted with $b=0$ ). The collective coordinates provide an economic means for parameterizing MES. Thus, the product states in the collective coordinates (which are MES in the particles coordinates) are conveniently given in terms of phase space like variables, $|q ; \ddot{0}\rangle_{r}|p ; 0\rangle_{c}$ (here $\ddot{0}$ and 0 denotes the bases mentioned above while $q$ and $p$ relates to the respective eigenvalues, $\omega^{q}$, $\omega^{p}$ with $\left.\omega=e^{i \frac{2 \pi}{d}} q, p=0,1, \ldots d-1\right)$. We thus have the $d^{2}$ dimensional Hilbert space imaged by the $d^{2}$ phase space like square whose points are specified by $(q, p)$ which are realized by the MES $|q ; \ddot{0}\rangle_{r}|p ; 0\rangle_{c}$.

We demonstrated that acting on the individual particles in a maximal entangled state leaves the particles unaffected while modifying the phase space like collective coordinates of the state.

We then considered "line states" $|L(m, b)\rangle$ which are defined (unnormalized) as the sum of MES along straight lines in the phase space like coordinated with $(q, p), q, p=0,1, \ldots d-1$. These are $p=b q-\mathrm{m}$ Mod $[d]$, for $b \neq \ddot{0}$ and $q=m^{\prime}$ for $b=\ddot{0}$. Thus, the parameter $(m, b)$ with $b=\ddot{0}, 0,1 . . d-1$ and $m=0,1, \ldots d-1$ define $d$ +1 families of parallel lines each containing $d$ phase space points $(q, p)$. Each family contains the whole $d^{2}$ phase space points once. We showed that each line state formed by adding all the product states (in the collective coordinates) parameterized by the phase space points $(q, p)$ lying on the line is a two particles product state, i.e. effecting a reversal of the Schmidt decomposition $[4,12]$ in giving a d-terms sum expansion of product states in terms of maximally entangled states (MES). These single particle product states turned out to be Mutual Unbiased Bases (MUB) states (reviewed in Appendix C). Thus, the study led to a novel scheme for obtaining MUB which are under intensive study in the fields of foundation of quantum mechanics, information theory and cryptography. This observation lends support to the attractive proposition initiated by [27] which identifies sum of paths (interpreted here as lines) with sum over MUB states. We showed that these line states are a product states in the particles coordinates. These single particle states turned out to be Mutually Unbiased States (MUB) which are discussed in Appendix C. Thus the study led to a novel scheme for obtaining MUB which are under intensive study in the fields of foundation of quantum mechanics, information theory and cryptography.

Acknowledgments I acknowledge with thanks numerous conversations and comments by my colleagues Professors A. Mann and J. Slawny.

\section{Appendix A: The "tilde" states}

As noted by [1] the $d$-dimensional Hilbert space is spanned by an orthonormal, computational basis (CB), $|n\rangle, \quad n=$ $0,1, \ldots d-1$ and all physical operators are functions of two operators, $\hat{Z}, \hat{X}$ defined by

$|n\rangle=|n+d\rangle, \quad \hat{Z}|n\rangle=\omega^{n}|n\rangle, \omega=e^{i \frac{2 \pi}{d}} ; \hat{X}|n\rangle=|n+1\rangle$.

Thus, we may label equivalently all $d$-dimensional Hilbert spaces each relative to its $\mathrm{CB}$ and choose the $\mathrm{CB}$ as real, i.e. we use the conventional reality of the scalar product of states in the CB: $\langle m \mid n\rangle^{*}=\langle m \mid n\rangle$. 
Consider an arbitrary state, $m$, in a basis $b:|m, b\rangle$ given in terms of its CB by

$|m ; b\rangle=\frac{1}{\sqrt{d}} \sum_{n=0}^{d+1} U_{m, n}^{b}|n\rangle$,

with $U$ a unitary operator. The state $|\tilde{m}, \tilde{b}\rangle$ is given by

$|\tilde{m} ; \tilde{b}\rangle=\frac{1}{\sqrt{d}} \sum_{n=0}^{d+1}\left(U_{m, n}^{b}\right)^{*}|n\rangle$,

is termed the "tilde" state associated with the state $|m, b\rangle$ [33]. Thus the relation between $|m, b\rangle$ and $|\tilde{m}, \tilde{b}\rangle$ is $\langle n \mid m, b\rangle=\langle n \mid \tilde{m}, \tilde{b}\rangle^{*}$.

The tilde states have the following property,

$|\mathcal{R}\rangle=\sum_{m}|m ; b\rangle_{1}|\tilde{m} ; \tilde{b}\rangle_{2}=\sum_{\alpha}|\alpha ; \beta\rangle_{1}|\tilde{\alpha} ; \tilde{\beta}\rangle \forall b, \beta$,

i.e., the state $|\mathcal{R}\rangle$ is "universal": i.e., independent of basis [29].

\section{Appendix B: State relabeling}

Given states enumeration $|n\rangle, n=0,1, \ldots d-1$ and the Schwinger operators, $Z$ the enumerating operator (with $|n\rangle$ its eigenfunctions) and $X$ their shifting operator $X|n\rangle=|n+1\rangle$. An arbitrary state, $\left|m ; b^{\prime}\right\rangle$ is specified in terms of $Z, X$ and $|n\rangle$. Thus labeling the state $\left|m ; b^{\prime}\right\rangle$, means that it is diagonalized (without degeneracies) by $F_{b^{\prime}}(X, Z)$ : $F_{b^{\prime}}(X, Z)\left|m ; b^{\prime}\right\rangle=\lambda(m)\left|m ; b^{\prime}\right\rangle$. Relabeling it by $|m+c ; b\rangle, c=0,1, \ldots d-1$, viz.

$\left|m ; b^{\prime}\right\rangle \rightarrow|m+c ; b\rangle, c=0,1, \ldots d-1$

where the latter is diagonalized by $F_{b}(X, Z)$ means that for transformed Schwinger operators $\bar{X}, \bar{Z}$ (and thence, transformed $\mathrm{CB}|n\rangle$, now are the eigenfunctions of $\bar{Z}$ ),

$F_{b^{\prime}}(X, Z)\left|m ; b^{\prime}\right\rangle=F_{b}(\bar{X}, \bar{Z})|m+c ; b\rangle$.

One readily sees that with $\left|m ; b^{\prime}\right\rangle=U|m+c ; b\rangle, \bar{X}=U X U^{\dagger} ; \bar{Z}=U Z U^{\dagger}$ and

$U=\sum_{n}\left|n ; b^{\prime}\right\rangle\langle b ; m+c|$

$\left|m ; b^{\prime}\right\rangle$ may be relabeled $|m+c ; b\rangle$. The relabeled states relate to the transformed operators.

The procedure is now illustrated for a simple case with $d=3$ where we wish to relabel the states of particle 2 as $\mathrm{CB}$ : Let the MES given as

$\left.\left|u(1,2\rangle=\frac{1}{\sqrt{3}} \sum\right| n\right\rangle_{1}\left|v_{n}\right\rangle_{2}$ 
with

$$
\left|v_{0}\right\rangle=\frac{1}{\sqrt{2}}(|0\rangle+|1\rangle) ;\left|v_{1}\right\rangle=\frac{1}{\sqrt{2}}(|0\rangle-|1\rangle) ;\left|v_{2}\right\rangle=|2\rangle .
$$

The desired relabeling is for the second particle state to be relabeled as CB state, i.e. eigenfunction of $\bar{Z}$, viz

$$
\left.\left|u(1,2\rangle=\frac{1}{\sqrt{3}} \sum\right| n\right\rangle_{1}\left|v_{n}\right\rangle_{2} \Rightarrow \frac{1}{\sqrt{3}} \sum|n\rangle_{1}|n\rangle_{2}
$$

The diagonalizing operator for the state marked for relabeling is (note: we assigned to it the desired spectrum, [2]),

$\hat{F}_{b^{\prime}}=\frac{1}{2}(|0\rangle+|1\rangle)\left(\langle 0|+\left\langle 1\left|+\frac{1}{2}(|0\rangle-|1\rangle) \omega\left(\langle 0|-\langle 1|+| 2\rangle \omega^{2}\langle 2|\right.\right.\right.\right.$.

The transformation operator,

$\hat{U}=\sum_{n}\left|v_{n}\right\rangle\langle n|$

Writing these operators explicitly in the (original) CB:

$\hat{F}_{b^{\prime}}=\left(\begin{array}{ccc}\frac{1+\omega}{2} & \frac{1-\omega}{2} & 0 \\ \frac{1-\omega}{2} & \frac{1+\omega}{2} & 0 \\ 0 & 0 & \omega^{2}\end{array}\right) \hat{U}=\left(\begin{array}{ccc}\frac{1}{\sqrt{2}} & \frac{1}{\sqrt{2}} & 0 \\ \frac{1}{\sqrt{2}} & -\frac{1}{\sqrt{2}} & 0 \\ 0 & 0 & 1\end{array}\right)$.

The state $|v\rangle$ (Eq. (26)) is now relabeled an eigenfunction of $\bar{Z}$, the "new" Schwinger enumerator operator. Thus $\hat{F}_{b^{\prime}} \rightarrow \bar{Z}$, while $|v\rangle \rightarrow|n\rangle$, i.e. $|v\rangle$ is relabeled $|n\rangle$.

Notes:

1. The relabeling is a basis, $b$, operation. Thus relabeling

$$
\left|m ; b^{\prime}\right\rangle \rightarrow|m+c ; b\rangle \Rightarrow\left|m^{\prime} ; b^{\prime}\right\rangle \rightarrow\left|m^{\prime}+c ; b\right\rangle .
$$

2. The procedure holds for all $1-1$ relation between the state to be relabeled ( $m$ in the analysis above) and its image ( $m+c$ in the analysis above). E.g. the image state could be the tilde state:

$$
\left|m ; b^{\prime}\right\rangle \rightarrow|\tilde{m} ; \tilde{b}\rangle .
$$

\section{Appendix C: Collective coordinates and collective bases}

The Hilbert space is spanned by the single particle computational bases, $|n\rangle_{1}\left|n^{\prime}\right\rangle_{2}$ (the subscripts denote the particles). These are eigenfunctions of $\hat{Z}_{i} i=1,2: \hat{Z}_{i}|n\rangle_{i}=\omega^{n}|n\rangle_{i}, \omega=e^{i \frac{2 \pi}{d}}$. Similarly $\hat{X}_{i}|n\rangle_{i}=|n+1\rangle_{i}, i=1,2$. Thus the exponents are modular variables. We now define our collective coordinates and collective operators (we remind the reader that the exponents are modular variables, e.g. $1 / 2 \bmod [d=7]=(d+1) / 2)=4)$ :

$\hat{Z}_{r} \equiv \hat{Z}_{1}^{1 / 2} \hat{Z}_{2}^{-1 / 2} ; \quad \bar{Z}_{c} \equiv \hat{Z}_{1}^{1 / 2} \hat{Z}_{2}^{1 / 2} \leftrightarrow \hat{Z}_{1}=\hat{Z}_{r} \hat{Z}_{c} ; \quad \hat{Z}_{2}=\hat{Z}_{r}^{-1} \hat{Z}_{c}$ 
and, in a similar manner,

$$
\hat{X}_{r} \equiv \hat{X}_{1} \hat{X}_{2}^{-1} ; \hat{X}_{c} \equiv \hat{X}_{1} \hat{X}_{2} \leftrightarrow \hat{X}_{1}=\hat{X}_{r}^{1 / 2} \hat{X}_{c}^{1 / 2}, \hat{X}_{2}=\hat{X}_{r}^{-1 / 2} \hat{X}_{c}^{1 / 2}
$$

We note that $\hat{Z}_{s}^{d}=\hat{X}_{s}^{d}=1$, and $\hat{X}_{s} \hat{Z}_{s}=\omega \hat{Z}_{s} \hat{X}_{s}, \quad s=r, c ; \hat{X}_{s} \hat{Z}_{s^{\prime}}=\hat{Z}_{s^{\prime}} \hat{X}_{s}, \quad s \neq s^{\prime} .\left|n_{1}\right\rangle\left|n_{2}\right\rangle$, the eigenfunctions of $\hat{Z}_{i}, i=1,2$, span the $d^{2}$ dimensional Hilbert space. The sets $\hat{Z}_{i}, \hat{X}_{i} ; i=1,2$ are algebraically complete in this space [1], i.e. every (non trivial) operator is a function of these operators. The eigenfunctions of $\hat{Z}_{q}$ are $\left|n_{c}, n_{r}\right\rangle$ with $\hat{Z}_{c}\left|n_{c}, n_{r}\right\rangle=\omega^{n_{c}}\left|n_{c}, n_{r}\right\rangle, \hat{Z}_{r}\left|n_{c}, n_{r}\right\rangle=\omega^{n_{r}}\left|n_{c}, n_{r}\right\rangle$. We note, e.g. [1], that $\left|n_{c}, n_{r}\right\rangle$ is equivalent to $\left|n_{c}\right\rangle\left|n_{r}\right\rangle$ when, as is the present case, the two sets, $\hat{Z}_{q}, \hat{X}_{q} ; q=c, r$ are compatible.

Clearly $|n\rangle_{r}\left|n^{\prime}\right\rangle_{c} ; n, n^{\prime}=0,1, . d-1$, is a $d^{2}$ orthonormal basis spanning the two $d$-dimensional particles' Hilbert space. We may consider their respective computation eigen bases (CB) and Fourier transform bases. To conform with previous notations [27-31] the CB is designated with $\ddot{0}$ while the eigenfunction of $\bar{X}$ (the Fourier transform of $\ddot{0}$ ) is designated with 0 :

$\hat{Z}_{s}|n ; \ddot{0}\rangle_{s}=\omega^{n}|n ; \ddot{0}\rangle_{s}, \quad \hat{X}_{s}|n ; 0\rangle=\omega^{n}|n ; 0\rangle ; \quad\left\langle n_{s} ; \ddot{0} \mid m_{s} ; 0\right\rangle=\omega^{-m_{s} n_{s}} . s=r, c$.

Note: we generally adopt the abbreviation $|n ; \ddot{0}\rangle \rightarrow|n\rangle$.

States in the particle coordinates may, clearly, be expressed in terms of the product states of the collective coordinates as both form a complete orthonormal basis that spans the two particles' Hilbert space,

$\left|n_{1}\right\rangle\left|n_{2}\right\rangle=\sum_{n_{c}, n_{r}}\left|n_{c}, n_{r}\right\rangle\left\langle n_{c}, n_{r} \mid n_{1}\right\rangle\left|n_{2}\right\rangle$.

The matrix element $\left\langle n_{c}, n_{r} \mid n_{1}\right\rangle\left|n_{2}\right\rangle$ is readily evaluated [29],

$\left\langle n_{1}, n_{2} \mid n_{r}, n_{c}\right\rangle=\delta_{n_{r},\left(n_{1}-n_{2}\right) / 2} \delta_{n_{c},\left(n_{1}+n_{2}\right) / 2}$.

Thus,

$\left|n_{c}\right\rangle_{c}\left|n_{r}\right\rangle_{r}=\left|n_{c}+n_{r}\right\rangle_{1}\left|n_{c}-n_{r}\right\rangle_{2}$

$\left|n_{1}\right\rangle_{1}\left|n_{2}\right\rangle_{2}=\left|\frac{n_{1}+n_{2}}{2}\right\rangle_{c}\left|\frac{n_{1}-n_{2}}{2}\right\rangle_{r}$.

We have then,

$\left|n_{r}, n_{c}\right\rangle \sim\left|n_{1}, n_{2}\right\rangle$, for $n_{r}=\left(n_{1}-n_{2}\right) / 2, n_{c}=\left(n_{1}+n_{2}\right) / 2 \rightleftarrows n_{1}=n_{r}+n_{c}, n_{2}=n_{c}-n_{r}$.

There are, of course, $d+1$ MUB bases for each of the collective modes. Here too, we adopt the notational simplification $b_{s} \rightarrow \ddot{0}_{s}, s=r, c$.

\section{Appendix D: Finite dimensional mutual unbiased bases, MUB, brief review}

In a finite, $d$-dimensional, Hilbert space two complete, orthonormal vectorial bases, $\mathcal{B}_{1}, \mathcal{B}_{2}$, are said to be MUB if and only if $\left(\mathcal{B}_{1} \neq \mathcal{B}_{2}\right)$

$\forall|u\rangle,|v\rangle \in \mathcal{B}_{1}, \mathcal{B}_{2} \operatorname{resp} ., \quad|\langle u \mid v\rangle|=1 / \sqrt{d}$.

The physical meaning of this is that knowledge that a system is in a particular state in one basis implies complete ignorance of its state in the other basis.

Ivanovich [15] proved that there are at most $d+1 \mathrm{MUB}$, pairwise, in a $d$-dimensional Hilbert space and gave explicit formulae for the $d+1$ bases in the case of $d=p$ (prime number). Wootters and Fields [18,19] constructed such $d+1$ bases for $d=p^{m}$ with $\mathrm{m}$ an integer. Variety of methods for construction of the $d+1$ bases for $d=p^{m}$ are now available $[16,17,20,26]$. Our present study is confined to $d=p \neq 2$. 
We now give explicitly the MUB states in conjunction with the algebraically complete operators [1,27] set: $\hat{Z}, \hat{X}$. Thus we label the d distinct states spanning the Hilbert space, termed the computational basis, by $|n\rangle, n=$ $0,1, . . d-1 ;|n+d\rangle=|n\rangle$

$\hat{Z}|n\rangle=\omega^{n}|n\rangle ; \hat{X}|n\rangle=|n+1\rangle, \omega=e^{i 2 \pi / d}$.

The $d$ states in each of the $d+1$ MUB $[20,27]$ are the states of computational basis and

$|m ; b\rangle=\frac{1}{\sqrt{d}} \sum_{0}^{d-1} \omega^{b n^{2}-n m}|n\rangle ; \quad b, m=0,1, . . d-1$.

Here the $d$ sets labeled by $b$ are the bases and the $m$ labels the states within a basis.

$\omega^{b} \hat{X} \hat{Z}^{2 b}|m ; b\rangle=\omega^{m}|m ; b\rangle$.

For later reference we refer to the computational basis (CB) by $b=\ddot{0}$. Thus we have $d+1$ MUB bases, $b=$ $\ddot{0}, 0,1, \ldots d-1$ with the total number of states $d(d+1)$ grouped in $d+1$ sets each of $\mathrm{d}$ states. We have of course,

$\left\langle m ; b \mid m^{\prime} ; b\right\rangle=\delta_{m, m^{\prime}} ; \quad\left|\left\langle m ; b \mid m^{\prime} ; b^{\prime}\right\rangle\right|=\frac{1}{\sqrt{d}}, \quad b \neq b^{\prime}$.

We mark states with double angular bracket $(|m ; b\rangle\rangle)$ to signify it to be an eigenfunction of (the relevant) unitary operator $\omega^{b} \hat{X} \hat{Z}^{2 b}$ for $\mathrm{b}=0,1, \ldots \mathrm{d}-1$ and of $\hat{Z}$ for $b=\ddot{0}$ (the latter signifies the computational basis) and thus a an MUB state, cf. [20].

\section{References}

1. Schwinger, J., Englert, B.-G. (eds.): Quantum mechanics: symbolism of atomic measurements, Chap. 1. Springer, Berlin, p. 69 (2001)

2. Schwinger, J.: Quantum uncertainty principle: no loopholes. Science 264, 1830-1831 (1994)

3. Braunstein, S.L., Mann, A., Revzen, M.: Maximal vilation of bell inequalities for mixed states. Phys. Rev. Lett. 68, 3259 (1992)

4. Peres, A.: Quantum theory: concepts and methods, Chap. 6. Kluwer, Dordrect (1995)

5. Bennett, C.H., Brassard, G., Crepeau, C., Jozsa, R., Peres, A., Wootters, W.L.: Teleporting an unknown quantum state via dual classical and einstein, podolsky and rosen channels. Phys. Rev. Lett. 70, 1895 (1993)

6. Clauser, J.F., Shimony, A.: Bell's theory: experimental tests and implications. Rep. Prog. Phys. 41, 1882 (1978)

7. Einstein, A., Podolsky, B., Rosen, N.: Can quantum-mechanical description of physical reality be considered complete? Phys. Rev. 47, 777 (1935)

8. Bell, J.S.: On the einstein, podolsky and rosen paradox. Physics 1, 195 (1964)

9. Lalö̈, F.: Do we really understand quantum mechanics? Strange correlations, paradoxes and theorems. Am. J. Phys. 69, 655 (2001)

10. Schroedinger, E.: Discussion of probability relations between separated systems. Math. Pro. Camb. Phil. Soc. 31, 555 (1935)

11. Fivel, D.: Remarable phase oscillations appearing in the lattice dynamics of eintein, podolsky and rosen states. Phys. Rev. Lett. 74, $835(1995)$

12. Fedorov, M.V., Miklin, N.I.: Schmidt modes and entanglement. Cont. Phys. 55(2), 94 (2014)

13. Saniga, M., Planat, M.: Viewing sets of mutually unbiased bases as arcs in finite projective planes. Chaos Solitons and Fractals 26, $1267(2005)$

14. Bennett, M.K.: Affine and Projective Geometry, Chap. 2. Wiley, New York, p. 26 (1995)

15. Ivanovic, I.D.: Geometrical description of quantal state determination. J. Phys. A 14, 3241 (1981)

16. Vourdas, A.: Phase space methods for finite quantum systems. Rep. Math. Phys. 40, 367 (1997)

17. Vourdas, A.: Quantum systems with finite hilbert space. Rep. Prog. Phys. 67, 267 (2004)

18. Wootters, W.K.: A wigner-function formulation of finite-state quantum mechanics. Ann. Phys. 176, 1 (1987)

19. Wootters, W.K., Fields, B.D.: Fields, optimal state-detrmination by mutually unbiased bases. Ann. Phys. 191, 363 (1989)

20. Bandyopadhyay, S., Boykin, P.O., Roychowdhury, V., Vatan, F.: A new proof for the existance of mutually unbiased bases. Algorithmica 34, 512 (2002) 
21. Gibbons, K.S., Hoffman, M.J., Wootters, W.K.: Discrete phase space based on finite fields. Phys. Rev. A 70, 062101 (2004)

22. Durt, T., Englert, B.-G., Bengtsson, I., Zyczkowski, K.: On mutually unbiased bases. Int. J. Quant. Info. 8, 535 (2010)

23. Barnett, S.M., Phoenix, S.T.D.: Phoenix, entropy as a measure of optical correlation. Phys. Rev. A. 40, 2404 (1989)

24. Barnett, S.M., Pegg, D.T., Phoenix, S.T.D.: Information, quantum correlations and communication. In: Tombasi, P., Walls, D.F. (eds.): Quantum Measurements in Optics, NATO ASI Series, vol. 282. Plenum Press, New York, p. 353 (1992)

25. Ban, M.: Completely entangled states and simultaneous eigenstates in finite dimensional space. Int. J. Phys. 47, 3267 (2008)

26. Klimov, A.B., Sanchez-Soto, L.L., de Guise, H.: Multycomplemetry operators via finite fourier transform. J. Phys. A: Math. Gen. 38, 2747 (2005)

27. Kalev, A., Mann, A., Revzen, M.: Choice of measurementas the signal. Phys. Rev. Lett. 110, 260502 (2013)

28. Kalev, A., Mann, A., Revzen, M.: Quantum mechanical retrodiction through an extended mean king problem. Eur. Phys. Lett. 104, 50008 (2013)

29. Revzen, M.: Maximally entangled states via mutually unbiased collective bases. Phys. Rev. A 81, 012113 (2010)

30. Revzen, M.: Geometrical underpinning of finite dimensional hilbert space. arXiv:1111.6446 [quant-ph] (2011)

31. Revzen, M.: Maximal entanglement collective coordinates and tracking the king. J. Phys. A 46, 075303 (2013)

32. Revzen, M.: Radon transform in finite hilbert space. Eur. Phys. Lett. 98, 10001 (2012)

33. Umezawa, H.: Advanced Field Theory, Chap. 7. AIP, NY, p. 160 (1993) 